\title{
La motivación de los estudiantes a participar en el Concurso Estatal de Aparatos y Experimentos de Física en Coahuila
}

\author{
Carlos Eduardo Rodríguez García \\ crodriguezgarcia@uadec.edu.mx \\ José Luis Fraga Almanza \\ josefraga@uadec.edu.mx \\ María Guadalupe Godina Cubillo \\ guadalupe.godina@uadec.edu.mx \\ José David Zaldívar Rojas \\ david.zaldivar@uadec.edu.mx \\ Roberto Constancio Torres Ramírez \\ roberto.torres@uadec.edu.mx \\ Manuel Antonio Torres Gomar \\ manuel.torres@uadec.edu.mx \\ Facultad de Ciencias Físico Matemáticas, Universidad Autónoma de Coahuila, Prol. \\ David Berlanga S/N, Edif. A. Unidad Camporredondo C.P.25000 \\ Saltillo Coahuila México \\ Christian Ivánn Rodriguez Garcia \\ stroktonbmp@hotmail.com \\ Centro de Estudios Tecnológicos Industrial y de Servicios CETIS 18, \\ Dirección General de Educación Tecnológica Industrial, Calle, Rio Rhin S/N Fracc. \\ Virreyes C.P. 21190 Mexicali Baja California México
}

\section{RESUMEN}

En este trabajo se realiza una breve encuesta dirigida a estudiantes de bachillerato del estado de Coahuila de Zaragoza para saber su motivación de participar en competiciones y/o Concurso de Física Experimental y Teórica. Esto en el marco de la realización del 7 Concurso Estatal de Aparatos de Experimentos de Física del estado de Coahuila el cual se llevó a cabo el pasado 23 de abril de 2021 en modalidad hibrida: presencial y en línea. Los resultados de la breve encuesta, indican que los estudiantes se motivan en este tipo de concursos debido al desarrollo de habilidades en la física, fomento de creatividad, y competitividad. Así mismo, manifiestan que la pandemia de Covid-19 si les ha afectado en el estudio de la Física, aunque una gran cantidad de 
estudiantes tiene pensado estudiar carreras relacionadas con la Ingeniería que involucran la Física como una de sus materias básicas. Finalmente, los estudiantes sugieren que los premios a este tipo de Concursos de Física debieran ser recomendaciones para ingresar en Universidades prestigiosas, dinero en efectivo o viajes científicos.

Palabras clave: Motivación; estudiantes; Concursos; Física; Estado de Coahuila 


\title{
The motivation of students to participate in the Experiments Physics and Apparatus Competition of Coahuila State
}

\begin{abstract}
We carried out in this work a brief survey to the high school students of the Coahuila's state. The main goal of such survey was figuring out the motivation of the students to participate in science competition fairs related with theoretical and experimental physics. The survey was achieved during the $7^{\text {th }}$ science competition fair of the state of Coahuila. This competition was carried out using the on-line and the face to face modes. According to the results in the survey, the students are motivated to participate in such event because they can increase their knowledge in physics and they also promote their creativity. Most of the students believe that the covid pandemic has affected their performance in the school to learn about physics. Despite this situation, they are considering a career in the field of physics or physics engineering. Finally, the students recommend that the award for the science fair should be cash, scientific travels or acceptance letters from prestigious universities to study physics.
\end{abstract}

Keywords: Motivation; Students; Competition; Physics; Coahuila state

Artículo recibido: 05 de Mayo 2021 Aceptado para publicación: 20 de Junio 2021 Correspondencia: crodriguezgarcia@uadec.edu.mx Conflictos de Interés: Ninguna que declarar 


\section{INTRODUCCIÓN}

Una manera de fomentar la creatividad en los jóvenes de bachillerato y de licenciatura es a través de las competiciones científicas (Esquivel Serrano, 2004). El potencial creativo puede estar relacionado con la realización de tareas, así como los recursos que se tengan para llevarla a cabo o que se puedan conseguir (Costa-Lobo y col, 2016). Para el aprendizaje de los conceptos, de cierta materia como la física, se requiere el estímulo de la creatividad (Velásquez Burgos B.M y col., 2010) que promueva en los estudiantes realizar experimentos, simulaciones, resolución de problemas, mapas mentales, etc. para el buen entendimiento de ciencias como la física o matemática.

La física es la ciencia que estudia la materia y sus interacciones mutuas, así como el movimiento de los cuerpos, los campos electromagnéticos, el comportamiento de la luz y la teoría atómica, por mencionar algunos de sus subsecciones de estudio (Alonso \& Finn, 1995, Serway, R. A. y Jewett, J. W. J., 2015). La física sigue como toda ciencia rigurosa, el método científico, para así explicar las propiedades y comportamiento de los fenómenos que observamos en la naturaleza. Este método científico podemos denotarlo como método experimental y es aplicable a todas las ciencias experimentales, depende de la cuidadosa observación y de la experimentación (Gutiérrez Muñoz J., 2007). La observación se desarrolla al examinar de forma detallada y critica los fenómenos, notando y analizando los factores que pueden influenciarlos. Así mismo, la experimentación radica en observar un fenómeno en condiciones preparados previamente y controlados con cuidado (Alonso \& Finn, 1995, Hewitt, 1995).

Una pregunta interesante de hacerse, es que motiva a los estudiantes de bachillerato en el estudio de la Física. Puede existir una motivación intrínseca, es decir, aquellos quienes que por interés interno les gusta el estudio de esta ciencia. Por lo regular, se va descubriendo paulatinamente, como aquel niño que se duerme con los libros sin poder entenderlos aún, tienes "hambre" de conocimiento se pudiera decir. Con relación al conocimiento que existe actualmente, nos encontramos en la era de la información, los alumnos hoy en día, así como los maestros están inundados de información (Pérez Salazar, 2011). Por lo que, lo esencial seria como distinguir entre un conocimiento verdadero y/o correcto entre la "paja" de información. No obstante, hay un conocimiento empírico al estudiar la física desde la óptica experimental, cuando un estudiante se involucra en un proyecto donde aplica los conocimientos de la física el 
aprendizaje se potencia. Además, si se involucra la competencia entre ellos se fomenta la creatividad y la formación de valores como el respeto por el trabajo del otro.

En gran parte de los estados de la república mexicana se realiza el Concurso de Aparatos y experimentos de física. El objetivo del Concurso es difundir y dar a conocer la Física Experimental entre los estudiantes de bachillerato y nivel superior premiándolos mediante una competencia por medio de categorías de Experimento, Aparato Didáctico y Aparato Tecnológico. A través de esta competencia también se tiene como objetivo motivar a los estudiantes, generar pensamiento de ingeniería y científico en ellos y con esto inducirlos al estudio de carreras como la Ingeniería Física, Matemáticas, Química y otras Ingenierías relacionadas a las Ciencias Básicas. Además, otro objetivo es lograr que los primeros lugares de cada categoría representen a cada estado en el Concurso Nacional de Aparatos y Experimentos de Física realizado por la Sociedad Mexicana de Física.

El público meta del Concurso son alumnos y alumnas de bachillerato y nivel superior de todos los semestres de entre 15 a 20 años de los distintos sistemas de bachillerato del estado de Coahuila. Así mismo, los docentes de Física y del área que los asesoran los cuales son de distintas edades.

La contribución del Concurso Estatal y Nacional de Aparatos y Experimentos de Física radica en el fomento de las vocaciones científicas en estudiantes de bachillerato hacia las ciencias físicas experimentales, acercamiento a la innovación por medio de un aparato didáctico, tecnológico o mediante el estudio de experimentos de física. Esto puede contribuir a que los estudiantes se motiven y puedan clarificar su orientación hacia las ciencias experimentales y las ingenierías.

\subsection{Un poco de historia del Concurso Estatal de Aparatos y Experimentos de Física}

A partir del año 2015 profesores de la Facultad de Ciencias Físico Matemáticas de la Universidad Autónoma de Coahuila (UAdeC) organizan el Concurso Estatal de Aparatos y Experimentos de Física en conjunto con la Sociedad Mexicana de Física. Esta competición originalmente dirigida a estudiantes de preparatoria desde hace un año se ha extendido al nivel de Licenciatura donde participan estudiantes de carreras como Ingeniería Física, Ingeniería Mecánica, Industrial, por mencionar algunas.

En las primeras versiones de 2015 a 2018 tuvimos una participación 360 estudiantes y profesores de bachilleratos de los municipios como Saltillo, Torreón, Piedras Negras, 
Parras de la Fuente, Acuña y Arteaga entre otros. Desde ese momento nos dimos cuenta el cuerpo de profesores de la Facultad que el concurso es muy importante para la motivación de los estudiantes en estudio de la Física experimental y tecnológica. En 2019, se tuvo la participación de 20 proyectos en donde estuvieron involucrados 60 alumnos y docentes de bachillerato. Ese año observamos una ligera disminución en la participación, esto fue atribuible a distintas razones, entre ellas que el concurso se cambió de fecha, usualmente se realizaba los meses de mayo y se movió al mes de agosto por cuestiones de presupuesto. En marzo de 2020, luego de la declaratoria de emergencia por la pandemia de Covid-19, los planes de la ejecución de la competición de llevarse a cabo en mayo se vinieron a bajo debido a la situación por los contagios de la enfermedad. Como es bien conocido por todos, las clases se empezaron a impartir de forma virtual en todos los niveles y entramos en un periodo distinto de realizar las actividades académicas. Esto mermó en animo de muchos estudiantes los cuales declinaron su participación en la edición 2020 del Concurso la cual se llevó a cabo en agosto de 2020 en modalidad en línea por medio de la plataforma Microsoft Teams la cual es provista por la Universidad Autónoma de Coahuila. El año de 2020, será un año que nos ha marcado a todos por las pérdidas, así mismo a estudiantes y docentes del nivel bachillerato y superior por ser un periodo con muchos retos de adaptación a las nuevas tecnologías de información y comunicación. Ese año el concurso convocó a estudiantes de bachillerato y nivel superior, con la finalidad de que no bajará más el ánimo dada la pandemia y que el espíritu del estudio de la física nos acompañara se decidió no cancelar el concurso. Se tuvo solo la participación de 20 estudiantes y 5 profesores en la competición (González A. y col., 2021). En cuanto al evento nacional, ese año no fue llevado a cabo.

El 7 Concurso de Aparatos y Experimentos de Física del estado de Coahuila de Zaragoza se llevó a cabo el pasado 23 de abril de 2021, se logró incrementar la participación teniendo 16 proyectos inscritos en las tres categorías, alrededor de 70 participantes entre estudiantes y asesores. El desarrollo y ejecución de la competencia fue en dos modalidades, en línea y presencial. El modo presencial se llevo a cabo en las instalaciones de la Facultad de Ciencias Físico Matemáticas de la Universidad Autónoma de Coahuila en la ciudad de Saltillo. La modalidad en línea fue utilizando la plataforma de Microsoft Teams, la cual se usa de forma institucional en la UAdeC. El 
poster electrónico del concurso se presenta en la Figura 1. Comúnmente se premia a los estudiantes con dispositivos como relojes inteligentes para los primeros lugares, audífonos de diadema para los segundos lugares y audífonos y libros científicos para los terceros lugares.

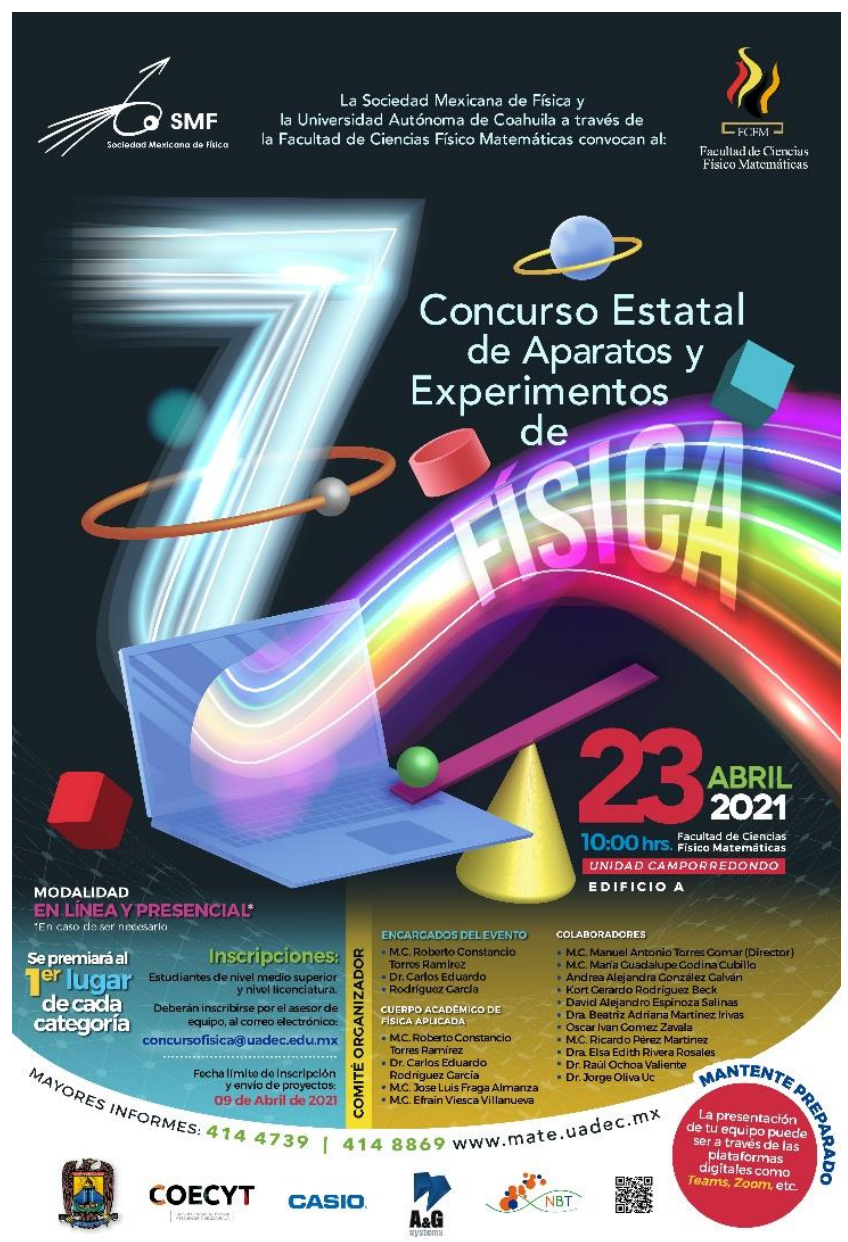

Figura 1: Imagen del póster para la difusión del 7 Concurso Estatal de Aparatos y Experimentos de Física del estado de Coahuila de Zaragoza.

Regresando a la pregunta inicial, que nos hicimos en cuanto a ¿qué es lo que motiva a los estudiantes en el estudio de la física, sobre todo en la física experimental? Además, como apoyo a las actividades del 7 Concurso Estatal de aparatos y Experimentos de Física este año se elaboró una breve encuesta, la cual se aplicó 200 estudiantes de todo el estado de Coahuila de Zaragoza, para conocer su opinión en cuanto a la motivación para el estudio de la física y saber cuál sería un aliciente para ellos de participar en el concurso además de su motivación intrínseca. 


\section{ESTRATEGIAS METODOLÓGICAS O MATERIALES Y MÉTODOS}

\subsection{Metodología}

Se diseñó una encuesta breve para apoyar a la ejecución del 7 Concurso Estatal de Aparatos y Experimentos de Física 2021, la breve encuesta la llamamos Motivación en el estudio de la Física, la cual incluyó las siguientes preguntas o ítems.

Nombre Completo:

Edad:

Nombre de la Escuela:

Semestre:

¿Qué tanto estas motivado(a) en el estudio de la Física?

¿Crees que la pandemia de Covid-19 ha afectado en cómo estudias Física?

¿Los concursos de Física Experimental o Teóricos te motivan en cuanto a:

a) Desarrollo de habilidades, b) desarrollo de creatividad, c) Competitividad, d) Gusto por exponer e) otros

¿Cómo crees que deben ser los premios de estos Concursos de Física Experimental y teóricos?

a) Dinero en efectico b) Viajes científicos, c) Recomendación para ingresar a Universidad prestigiosa

¿Qué carrera deseas estudiar?

Esta encuesta se implementó en Google Forms y se distribuyó con docentes de las escuelas de bachillerato en el estado de Coahuila de Zaragoza para que ellos las aplicarán en sus clases virtuales. La encuesta se aplicó a 200 alumnos de bachillerato del estado de Coahuila utilizando Google Forms, la recolección de las respuestas de esta encuesta fue durante el periodo de enero hasta marzo de 2021. La encuesta se realizó a estudiantes de bachillerato de las ciudades más importantes del estado de Coahuila, las cuales fueron: Saltillo, Torreón, Monclova, Piedras Negras y Ramos Arizpe.

Los resultados se graficaron y se interpretaron logrando inferir la motivación de los estudiantes a participar en el Concurso Estatal de Aparatos y Experimentos de Física en el estado de Coahuila.

\section{RESULTADOS Y DISCUSIÓN}

La media de edad de los estudiantes encuestados de bachillerato fue de 16.7 años, siendo la mediana y moda 17 años esto dado que la mayoría de los alumnos se 
encuentran en 4to y 6to semestre de bachillerato. En la Figura 2a), se presenta la distribución de edades de los alumnos en forma de histograma, se puede notar que la mayoría de los estudiantes encuestados tienen entre 16 y 17 años. Además, en la Figura 2b) se muestra el gráfico de pastel de los porcentajes correspondientes a las ciudades de origen del estado de Coahuila de los estudiantes encuestados, ligeramente más de la mitad de los encuestados son de la ciudad de Saltillo (55.5\%), en segundo orden fueron los encuestados de la ciudad de Piedras Negras (32\%) y el resto de las ciudades de Torreón (7\%), Monclova (5\%) y Ramos Arizpe (0.5\%), todos municipios importantes de Coahuila. Algunas de los bachilleratos fueron, la Escuela de Bachilleres Ateneo Fuente, Cbtis \#34 Piedras Negras, Colegio Cervantes de Torreón, Universidad Carolina campus Saltillo, Conalep Saltillo 2 y Colegio Ignacio Zaragoza.
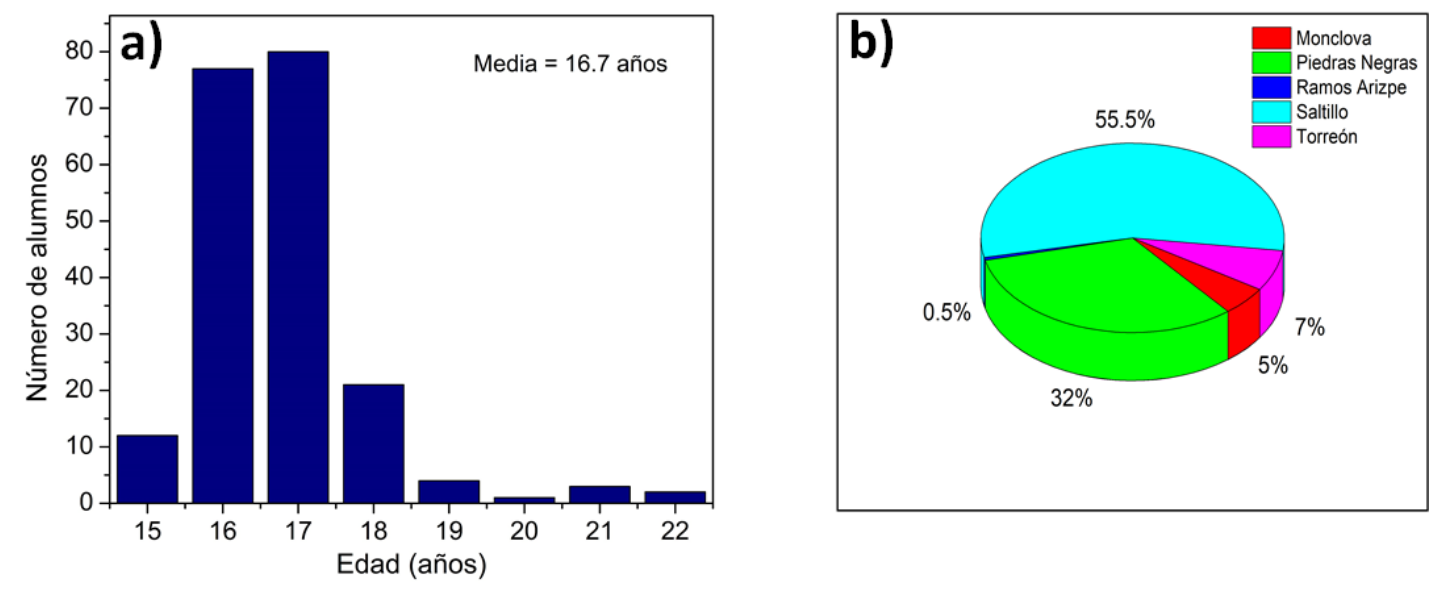

Figura 2: a) Histograma de distribución de edades de estudiantes de bachillerato encuestados por edades. b) Gráfico de pastel con indicando el porcentaje del lugar de residencia de los estudiantes al aplicar la encuesta.

La encuesta mencionada se centró más en estudiantes que ya habían cursado al menos una materia de Física, por esa razón, la mayor cantidad de encuestados, es decir un 89\% se ubican del 4to al 6to semestre tal como se puede observar en la gráfica de columnas de la Figura 3, esto corresponde a 178 alumnos. El resto fueron de los semestres de 1ero a 3ero, esto es 22 alumnos, recordemos que la población de muestra fue de 200 estudiantes. 


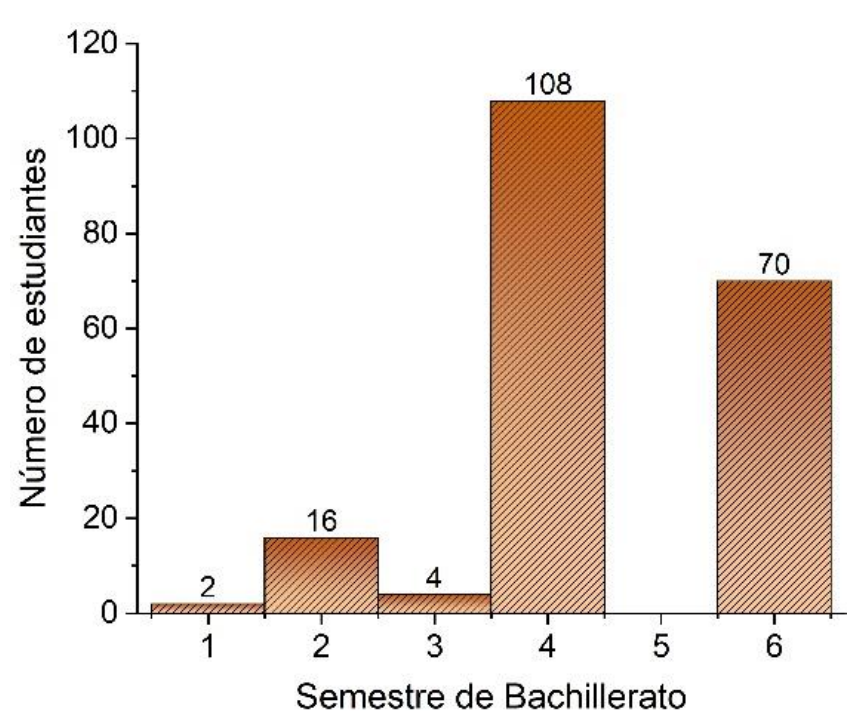

Figura 3: Gráfica de barras que indica el número de estudiantes encuestados como función del semestre que actualmente está cursando.

Dos de las preguntas planteadas a los estudiantes, y qué para todos los docentes es interesante saber, es ¿Qué tanto estás motivado (a) en el estudio de la Física? y, ¿Los Concursos de Física experimental y o teóricos me motivan en cuando a:? En la primera cuestión, se les dio las cuatro opciones a elegir a los alumnos desde 1) Nada, 2) Poco, 3) Regular y 4) Mucho. Para la segunda pregunta se les dieron las siguientes opciones, los concursos me motivan a fomentar mí: 1) Competitividad, 2) Creatividad, 3 ) Desarrollo de habilidades en física y 4) Gusto por exponer. Estás alternativas de elección fueron puestas según el criterio de los organizadores el cual resultó de diálogos con participantes del Concurso Estatal de Aparatos y Experimentos de Física en el estado de Coahuila en el periodo del 2015 al 2019. En la Figura 4 a) se muestra el gráfico de pastel sobre las respuestas a la primera pregunta ¿Qué tanto estás motivado (a) en el estudio de la Física? hecha a los alumnos, se puede observar que un $60.5 \%$ esta motivado de forma regular, digamos un poco menos de totalmente motivado según el criterio. Hay un $25.5 \%$ qué esta muy motivado en el estudio de la física, lo cual es muy bueno. Un $12 \%$ y un $2 \%$ está poco o nada motivado en el estudio de la física, esto puede ser a diversas razones vocacionales, quizá no estudiarán una carrera afín a la física en un futuro. Como pequeña conclusión, se puede decir que la mayoría de los estudiantes encuestados están motivados en cierto modo en el estudio de esta ciencia compleja para algunos que es la física (Arandia E y col., 2016, Méndez Coca D., 2015). Respecto a la segunda pregunta hecha a los alumnos: ¿Los Concursos de Física 
experimental y o teóricos me motivan en cuando a:? Los resultados de esta pregunta se exhiben en la figura 4 b) en gráfico de pastel. La generalidad de los estudiantes (63\%) contestó que este tipo de competiciones fomentan su desarrollo de habilidades en la física, en un segundo lugar (28.5\%) contestaron que promueve su creatividad, el resto (8.5\%) de los estudiantes respondió que promueve la competitividad y el gusto por exponer.
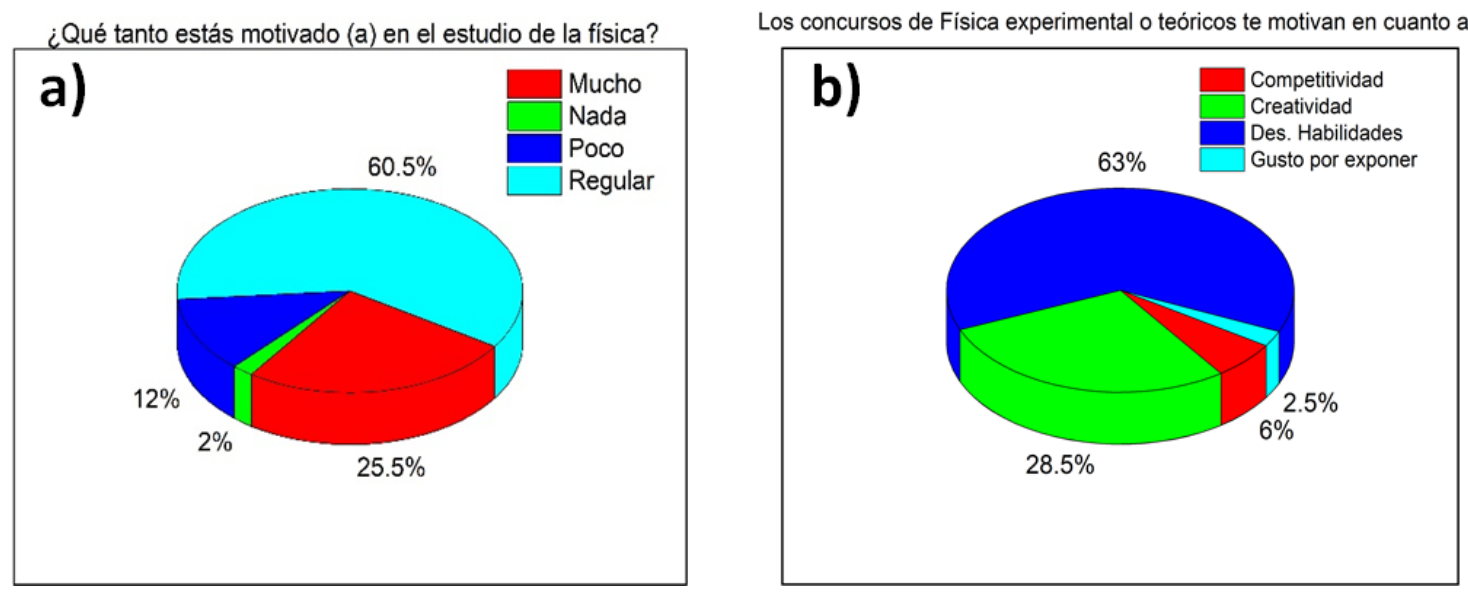

Figura 4: a) Gráfico de pastel que ilustra las respuestas de los estudiantes a la pregunta sobre el nivel de motivación en el estudio de la física b) Gráfica de pastel sobre las respuestas elegidas por los alumnos de la pregunta sobre la motivación y lo que aportan los Concursos de física experimental y teórica.

Otras preguntas importantes del breve sondeo de la encuesta, fueron las siguientes, ¿Crees que la pandemia de Covid-19 te ha afectado en cómo estudias Física? y ¿Cuáles crees que deben ser los premios de los concursos de física experimental y teóricos? En la Figura 5a) y 5 b) se presentan los gráficos con las opciones elegidas por los estudiantes. Primero, en cuanto a sobre cómo ha afectado la pandemia de Covid-19 en el estudio de la física por los estudiantes, un $38.5 \%$ contestó que le ha afectado mucho estudiar física, seguido de un $35.5 \%$ que consideró que le ha perjudicado de manera regular, no obstante, al sumar estos porcentajes, nos da un $74 \%$ que representan estudiantes que de cierta manera han sido damnificados por la pandemia de Covid-19, esto ha sido reportado por Liang L. y Col. en 2020. Además, otros estudios sugieren que la pandemia de Covid-19 ha impactado en la motivación del estudio en otras materias (Urzua M. y col., 2020, Espinoza F.Y. y col., 2020). Las otras respuestas con los porcentajes menores de $19.5 \%$ y $6.5 \%$, fueron de alumnos que contestaron que les afectó poco y nada, respectivamente, la situación de la enfermedad de coronavirus. Es 
interesante, analizar la respuesta a la pregunta: ¿Cuáles crees que deben ser los premios de los concursos de Física Experimental y Teóricos?, ya que esto incide fundamentalmente con su motivación, satisfacción y recompensa en el estudio académico de esta ciencia. La opción mayoritariamente elegida fue que el premio debería ser una recomendación para ingresar a la Universidad, luego en igual número de elección fueron las respuestas viaje científico y dinero en efectivo, por último, encontramos las opciones de libros de física y otros. El resultado de la opción mayoritaria hace inferir que la mayoría de los alumnos están preocupados por el ingreso al bachillerato y suponemos que, por ser admitidos en una Universidad de prestigio, esto podría ser aprovechado por los bachilleratos al mejorar las opciones de terminaciones vocacionales con énfasis en la física.
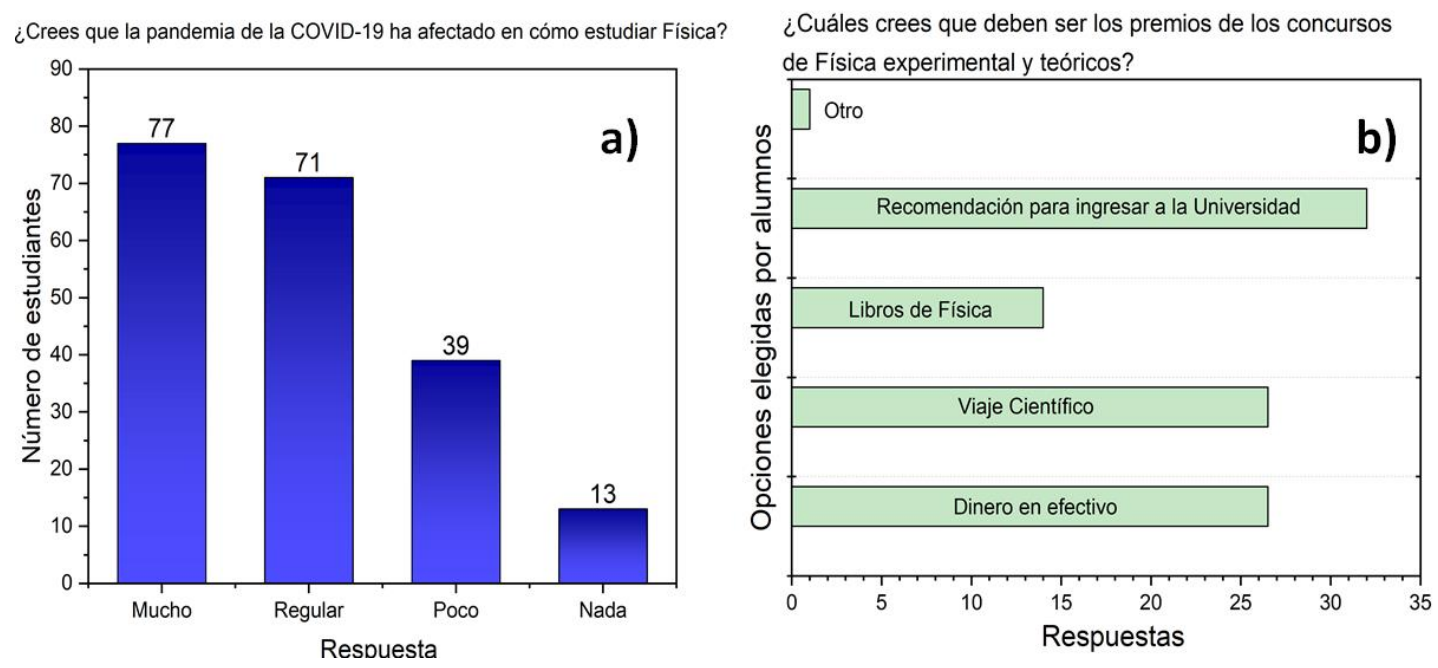

Figura 5: a) Histograma que muestra las respuestas de los alumnos a la pregunta relacionada a la afectación de la pandemia de Covid-19 en el estudio de la física. b)

Gráfico de columnas que muestra las opciones elegidas por los estudiantes con relación a lo que ellos piensan que deberían ser los premios en los concursos de física experimental y teóricos.

Algo que nos concierne como docentes de la Universidad Autónoma de Coahuila, y por tener la carrera de Ingeniería Física en la Facultad de Ciencia Físico Matemáticas, es si los estudiantes de bachillerato conocen o han escuchado nuestra carrera en el estado de Coahuila, o la existencia de la misma. La respuesta de si conocen o no, se ilustra en el gráfico de pastel de la Figura 6. Es sorprendente notar que un alto porcentaje $82.5 \%$ de los estudiantes encuestados no conocen la existencia de la carrera de Ingeniería Física de la Universidad Autónoma de Coahuila. Esto nos sugiere que la carrera de Ingeniería 
Física y el perfil de la misma no ha sido difundidos de forma adecuados, o quizá se requiera un cambio de estrategia, Aunque, es confortable el resultado de que un $17.5 \%$ conoce la carrera de Ingeniería Física, hay arduo trabajo por realizar en cuanto a la publicidad de la misma.

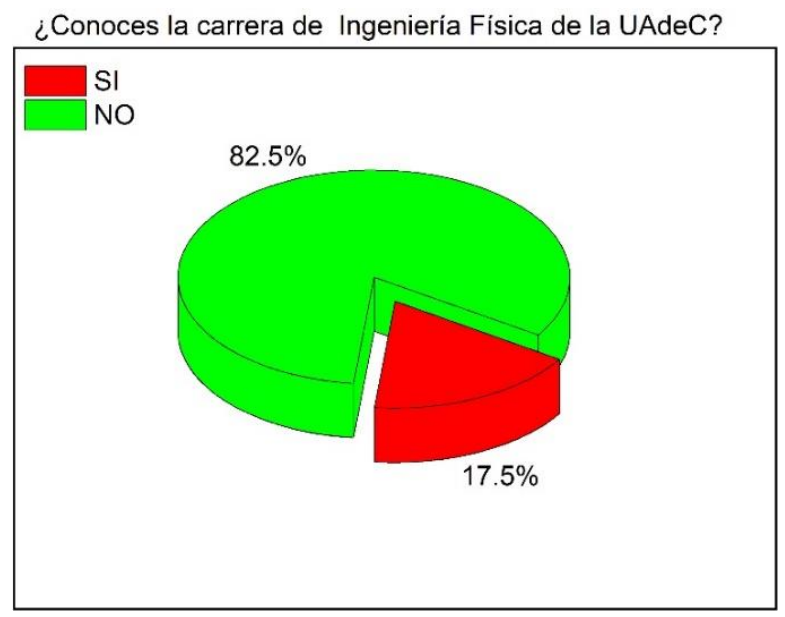

Figura 6: Gráfico de pastel que presenta los resultados sobre el conocimiento de la existencia de la carrera de Ingeniería de Física en el Estado de Coahuila de Zaragoza.

Como parte casi final de la breve encuesta a los estudiantes de bachilleratos, se les cuestionó sobre qué carrera tienen pensado estudiar en el futuro cercano. En la Figura 7 se presentan los resultados en gráfica columnar donde se agruparon las opciones contestadas por los estudiantes. Es relevante notar que en esta pregunta no se les dieron opciones de respuesta, simplemente ellos escribieron la opción, estas elecciones pueden estar relacionadas con la satisfacción que muestran los egresados de esas carreras en sus trabajos (Wolniak, G. C. y col., 2005). Así que, procesando los resultados se clasificaron en cuanto a las frecuencias de las mismas opciones escritas por los estudiantes y carreras con alta similitud. Algo alentador es que una gran cantidad de estudiantes (81) estudiará alguna Ingeniería, entre las que colocaron Ing. Física, electrónica, mecánica, mecatrónica, química, automotriz, sistemas, industrial, etc. Esto puede ser bien interpretado dadas las características del estado de Coahuila de Zaragoza que se destaca como un estado mayoritariamente industrial, contando con las zonas industriales de Ramos Arizpe, Saltillo, La Laguna-Torreón, y en la frontera en Piedras Negras. Las segundas carreras más elegidas fueron Medicina (22), Informática (18), Administración y Finanza (16) s y Diseño Digital, Gráfico y Artes (15). Finalmente, el resto de los estudiantes (71) escribió que su elección son las carreras de Criminología, 
Psicología, Nutrición, Derecho, Educación, Arquitectura y Gastronomía, y solo 7 estudiantes aún no han elegido qué estudiar.

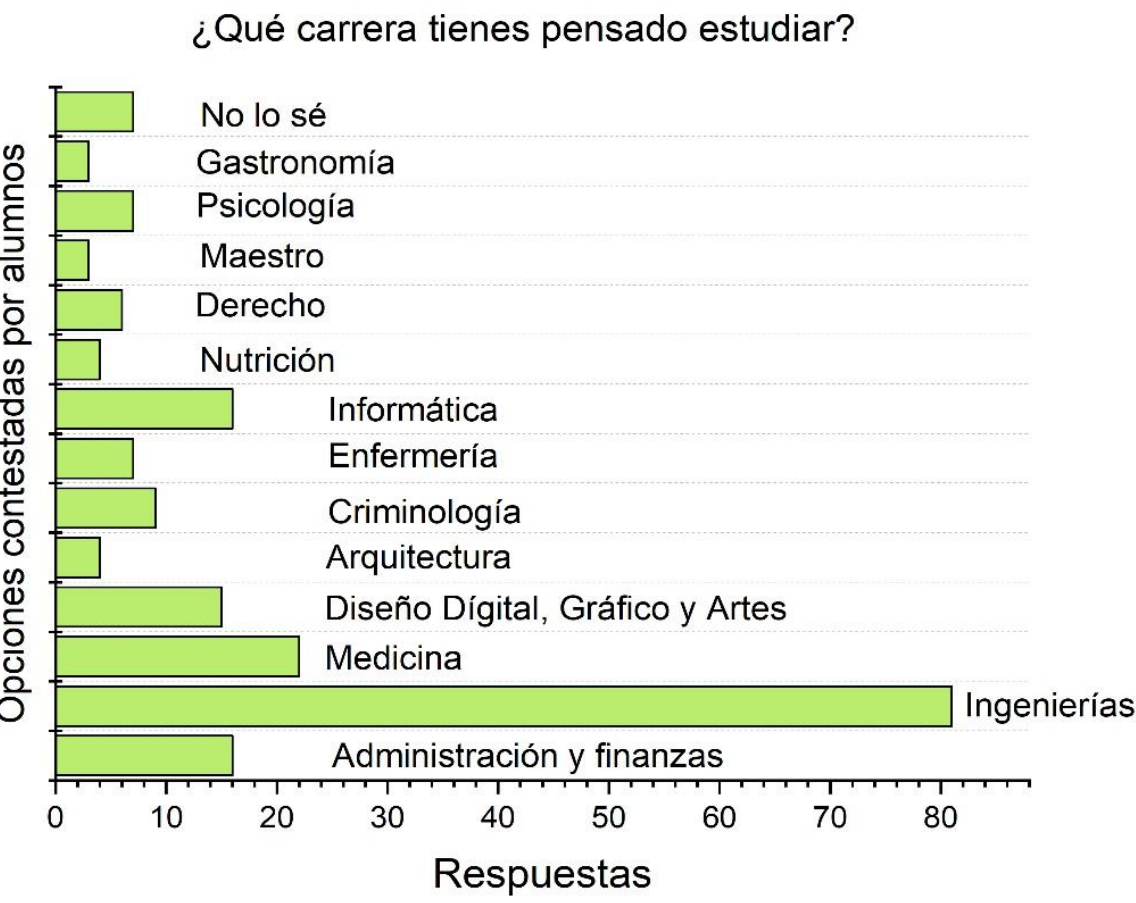

Figura 7: Gráfica de columnas horizontales que expone las opciones de las carreras que los estudiantes de bachillerato encuestados desean ingresar en un corto plazo.

\section{CONCLUSIONES}

Como conclusiones finales de este breve estudio sobre la motivación de los estudiantes de bachillerato en el estudio de la física, previamente efectuado al 7 Concurso Estatal de Aparatos y Experimentos de Física del Estado de Coahuila de Zaragoza destacamos las siguientes:

Es importante la motivación de los estudiantes de bachillerato en nivel medio superior ya que fomenta las vocaciones científicas y de ingeniería. Como se vio en la breve encuesta una gran cantidad de alumnos desean estudiar una carrera relacionada con la ingeniería. Algo significativo que se descubrió es que los estudiantes encuestados (200), en su mayoría ya tienen una decisión para estudiar, y que esta cantidad de estudiantes es una muestra representativa de los alumnos de Coahuila en bachillerato próximos a egresar y por elegir carrera. En cuanto, al estudio de la física podríamos afirmar que el estudiante común si le ha afectado la pandemia, esto puede ser multifactorial, aunque un factor esencial puede ser que las clases desde marzo del año 
2020 se han estado realizando en modalidad virtual, otro factor podríamos asociarlo a que por lo regular cada familia ha tenido un caso de enfermedad por covid-19, familiares cercanos o lejanos.

Otra conclusión a resaltar es que los Concursos de Física Experimental o Teórica fomenta el desarrollo de habilidades en la física, la creatividad y la competitividad, lo cual puede estar relacionado con la elección de carrera o formación de vocaciones en el estado de Coahuila que es un estado fundamentalmente industrial y manufacturero automotriz. También, un área de oportunidad que nos concierne como docentes es la difusión del perfil de la carrera de Ingeniero Físico en el estado de Coahuila, ya que una gran cantidad de estudiantes encuestados de bachillerato desconoce sobre la existencia de la misma.

En una perspectiva general, recomendamos seguir realizando el Concurso Estatal de Aparatos y Experimentos de Física, aún cuando las modalidades puedan ser en línea dada la situación de covid-19, ya que no hay nada más motivante para los alumnos que cuando un grupo de docentes trabaja por ellos y para ellos, en nuestra experiencia si hemos visto una participación moderada y esperamos que el Concurso Nacional de Aparatos y Experimentos de Física 2021 se lleve a cabo a pesar de la pandemia de covid-19.

Agradecimientos: Los autores de este articulo agradecemos el apoyo del Consejo Estatal de Ciencia y Tecnología (COECYT) del estado de Coahuila de Zaragoza dentro del programa FONCYT. Además, los autores agradecen el soporte de la Dirección de Investigación y Posgrado de la Universidad Autónoma de Coahuila.

\section{LISTA DE REFERENCIAS}

Alonso M. y Finn E. (1995). Física, México: Addison-Wesley Iberoamericana.

Arandia E., Zuza K. y Guisasola J. (2016). Actitudes y motivaciones de los estudiantes de ciencias en Bachillerato y Universidad hacia el aprendizaje de la Física. Revista Eureka sobre Enseñanza y Divulgación de las Ciencias, 558-573.

Costa-Lobo C., Perez Nieto, M.A., Castillo-Parra G., Vestena Carla L.B., CabreraCuevas J. (2016). Propuestas Psicoeducativas para promover la Creatividad en Contextos Educativos., eduPsykhé, 111-141. 
Espinosa F. Y., Mesa Trujillo D., Díaz Castro Y., Caraballo García L., Mesa Landín M.A. (2020), Estudio del impacto psicológico de la COVID-19 en estudiantes de Ciencias Médicas, Los Palacios Revista Cubana de Salud Pública, 1-17.

Esquivel Serrano M.T. (2004). Creatividad: Definiciones, Antecedentes y Aportaciones., Revista Digital Universitaria, 1067-6079.

González, A., Rodríguez, K., Rodríguez, C., Torres, R., Fraga, L. y Viesca, E. (2021). Concurso Estatal de Aparatos y Experimentos de Física 2020 en el contexto de la pandemia de Covid-19. Tlahuizcalli, 14-20.

Gutiérrez Muñoz J. (2007), La Física, Ciencia Teórica y Experimental, Vivat Academia, 24-41.

Hewitt, P. G. (1999). Física Conceptual (1a. ed.). México: Addison Wesley Longman.

Liang, L., Ren, H. Cao, R. Hu Y., Q. Zeying, Li C., Mei S. (2020). Effect of COVID19 on Youth Mental Health. Psychiatric Quarterly, 841-852.

Méndez Coca, D. (2015). Estudio de las motivaciones de los estudiantes de secundaria de física y química y la influencia de las metodologías de enseñanza en su interés. Educación XX1, 215-235.

Pérez Salazar G. (2011)., La Web 2.0 y la sociedad de la información., Revista mexicana de ciencias políticas y sociales. 57-68.

Serway, R. A., y Jewett, J. W. J. (2015). Física para ciencias e ingeniería (9a. ed.). México: Cengage Learning.

Urzúa, M., Rodríguez, D., Valencia, M., \& Ruiz, R. (2020). Aprender ciencias experimentales mediante TIC en tiempos de covid-19: percepción del estudiantado. Praxis \& Saber, e11447, 1-21.

Velásquez Burgos B.M., Remolina de Cleves N., Calle Márquez M.G. (2010), La creatividad como práctica para el desarrollo del cerebro total, Tabula Rasa. 321338.

Wolniak, G. C. y Pascarella, E. T. (2005). The effects of college major and job field congruence on job satisfaction. Journal of Vocational Behavior, 233-251. 